\title{
Approximately cubic functional equations and cubic multipliers
}

\author{
Abasalt Bodaghi ${ }^{1 *}$, Idham Arif Alias ${ }^{2}$ and Mohammad Hossein Ghahramani ${ }^{1}$
}

* Correspondence: abasalt. bodaghi@gmail.com

'Department of Mathematics, Garmsar Branch, Islamic Azad University, Garmsar, Iran

Full list of author information is available at the end of the article

\section{Abstract}

In this paper, we prove the Hyers-Ulam stability and the superstability for cubic functional equation by using the fixed point alternative theorem. As a consequence, we show that the cubic multipliers are superstable under some conditions.

2000 Mathematics Subject Classification: 39B82; 39852.

Keywords: cubic functional equation, multiplier, Hyers-Ulam stability, Superstability

\section{Introduction}

The stability problem for functional equations is related to the following question originated by Ulam [1] in 1940, concerning the stability of group homomorphisms: Let $\left(G_{1}\right.$, .) be a group and let $\left(G_{2},{ }^{*}\right)$ be a metric group with the metric $d(.$, .). Given $\varepsilon>0$, does there exist $\delta>0$ such that, if a mapping $h: G_{1} \rightarrow G_{2}$ satisfies the inequality $d(h(x, y), h(x)$ * $h(y))<\delta$ for all $x, y \in G_{1}$, then there exists a homomorphism $H: G_{1} \rightarrow G_{2}$ with $d(h(x)$, $H(x))<\varepsilon$ for all $x \in G_{1}$ ?

In 1941, Hyers [2] gave a first affirmative answer to the question of Ulam for Banach spaces. Later, Rassias in [3] provided a remarkable generalization of the Hyers' result by allowing the Cauchy difference to be bounded for the first time, in the subject of functional equations and inequalities. Găvruta then generalized the Rassias' result in [4] for the unbounded Cauchy difference.

The functional equation

$$
f(x+y)+f(x-y)=2 f(x)+2 f(y)
$$

is called quadratic functional equation. Also, every solution (for example $f(x)=a x^{2}$ ) of functional Equation (1.1) is said to be a quadratic mapping. A Hyers-Ulam stability problem for the quadratic functional equation was proved by Skof [5] for mappings $f: \mathcal{X} \rightarrow \mathcal{Y}$, where $\mathcal{X}$ is a normed space and $\mathcal{Y}$ is a Banach space. Cholewa [6] noticed that the theorem of Skof is still true if the relevant domain $\mathcal{X}$ is replaced by an abelian group. In [7] Czerwik proved the Hyers-Ulam-Rassias stability of the quadratic functional equation. Several functional equations have been extensively investigated by a number of authors (for instances, [8-10]).

Jun and Kim [11] introduced the functional equation

$$
f(2 x+y)+f(2 x-y)=2 f(x+y)+2 f(x-y)+12 f(x)
$$

(c) 2011 Bodaghi et al; licensee Springer. This is an Open Access article distributed under the terms of the Creative Commons Attribution License (http://creativecommons.org/licenses/by/2.0), which permits unrestricted use, distribution, and reproduction in any medium, provided the original work is properly cited. 
which is somewhat different from (1.1). It is easy to see that function $f(x)=a x^{3}$ is a solution of (1.2). Thus, it is natural that Equation (1.2) is called a cubic functional equation and every solution of this cubic functional equation is said to be a cubic function. One year after that, they solved the generalized Hyers-Ulam-Rassias stability of a cubic functional equation $f(x+2 y)+f(x-2 y)+6 f(x)=f(x+y)+4 f(x-y)$ in Jun and Kim [12]. Since then, a number of authors (for details see $[13,14]$ ) proved the stability problems for cubic functional equation.

Recently, Bodaghi et al in [15] proved the superstability of quadratic double centralizers and of quadratic multipliers on Banach algebras by fixed point methods. Also, the stability and the superstability of cubic double centralizers of Banach algebras which are strongly without order had been established in Eshaghi Gordji et al. [16].

In this paper, we remove the condition strongly without order and investigate the generalized Hyers-Ulam-Rassias stability and the superstability by using the alternative fixed point for cubic functional Equation (1.2) and their correspondent cubic multipliers.

\section{Stability of cubic function equations}

Throughout this section, $X$ is a normed vector space and $Y$ is a Banach space. For the given mapping $f: X \rightarrow Y$, we consider

$$
D f(x, y):=f(2 x+y)+f(2 x-y)-2 f(x+y)-2 f(x-y)-12 f(x)
$$

for all $x, y\lfloor X$.

We need the following known fixed point theorem, which is useful for our goals (an extension of the result was given in Turinici [17]).

Theorem 2.1. (The fixed point alternative [18]) Suppose that $(\Omega, d)$ a complete generalized metric space and let $\mathcal{J}: \Omega \rightarrow \Omega$ be a strictly contractive mapping with Lipschitz constant $L<1$. Then for each element $x \in \Omega$, either $d\left(\mathcal{J}^{n} x, \mathcal{J}^{n+1} x\right)=\infty$ for all $n \geq 0$, or there exists a natural number $n_{0}$ such that:

(i) $d\left(\mathcal{J}^{n} x, \mathcal{J}^{n+1} x\right)<\infty$ for all $n \geq n_{0}$;

(ii) the sequence $\left\{\mathcal{J}^{n} x\right\}$ is convergent to a fixed point $y^{*}$ of $\mathcal{J}$;

(iii) $y^{*}$ is the unique fixed point of $\mathcal{J}$ in the set

$$
\Lambda=\left\{y \in \Omega: d\left(\mathcal{J}^{n_{0}} x, y\right)<\infty\right\}
$$

(iv) $d\left(y, y^{*}\right) \leq \frac{1}{1-L} d(y, \mathcal{J} y)$ for all $y \in \Lambda$.

Theorem 2.2. Let $f: X \rightarrow Y$ be a mapping with $f(0)=0$, and let $\psi: X \times X \rightarrow[0, \infty)$ be a function satisfying

$$
\lim _{n \rightarrow \infty} \frac{\psi\left(2^{n} x, 2^{n} y\right)}{8^{n}}=0
$$

and

$$
\|D f(x, y)\| \leq \psi(x, y)
$$


for all $x, y \in X$. If there exists $L \in(0,1)$ such that

$$
\psi(2 x, 0) \leq 8 L \psi(x, 0)
$$

for all $x \in X$, then there exists a unique cubic mapping $C: X \rightarrow Y$ such that

$$
\|f(x)-C(x)\| \leq \frac{L}{2(1-L)} \psi(x, 0)
$$

for all $x \in X$.

Proof: We consider the set $\Omega:=\{g: X \rightarrow Y \mid g(0)=0\}$ and introduce the generalized metric on $\Omega$ as follows:

$$
d\left(g_{1}, g_{2}\right):=\inf \left\{C \in(0, \infty):\left\|g_{1}(x)-g_{2}(x)\right\| \leq C \psi(x, 0) \text { for all } x \in X\right\}
$$

if there exists such constant $C$, and $d\left(g_{1}, g_{2}\right)=\infty$, otherwise. One can prove that the metric space $(\Omega, d)$ is complete. Now, we define the mapping $\mathcal{J}: \Omega \rightarrow \Omega$ by

$$
\mathcal{J} g(x)=\frac{1}{8} g(2 x), \quad(x \in X) .
$$

If $g_{1}, g_{2} \in \Omega$ such that $d\left(g_{1}, g_{2}\right)<C$, by definition of $d$ and $\mathcal{J}$, we have

$$
\left\|\frac{1}{8} g_{1}(2 x)-\frac{1}{8} g_{2}(2 x)\right\| \leq \frac{1}{8} C \psi(2 x, 0)
$$

for all $x \in X$. By using (2.3), we get

$$
\left\|\frac{1}{8} g_{1}(2 x)-\frac{1}{8} g_{2}(2 x)\right\| \leq C L \psi(x, 0)
$$

for all $x \in X$. The above inequality shows that $d\left(\mathcal{J} g_{1}, \mathcal{J} g_{2}\right) \leq L d\left(g_{1}, g_{2}\right)$ for all $g_{1}, g_{2}$ $\in \Omega$. Hence, $\mathcal{J}$ is a strictly contractive mapping on $\Omega$ with Lipschitz constant $L$. Putting $y=0$ in (2.2), using (2.3), and dividing both sides of the resulting inequality by 16 , we have

$$
\left\|\frac{1}{8} f(2 x)-f(x)\right\| \leq \frac{1}{16} \psi(x, 0) \leq \frac{1}{2} L \psi\left(\frac{x}{2}, 0\right)
$$

for all $x \in X$. Thus, $d(f, \mathcal{J} f) \leq \frac{L}{2}<\infty$. By Theorem 2.1, the sequence $\left\{\mathcal{J}^{n} f\right\}$ converges to a fixed point $C: X \rightarrow Y$ in the set $\Omega_{1}=\{g \in \Omega ; d(f, g)<\infty\}$, that is

$$
C(x)=\lim _{n \rightarrow \infty} \frac{f\left(2^{n} x\right)}{8^{n}}
$$

for all $x \in X$. By Theorem 2.1, we have

$$
d(f, C) \leq \frac{d(f, \mathcal{J} f)}{1-L} \leq \frac{L}{2(1-L)} .
$$

It follows from (2.6) that (2.4) holds for all $x \in X$. Substituting $x, y$ by $2^{n} x, 2^{n} y$ in (2.2), respectively, and applying (2.1) and (2.5), we have

$$
\begin{aligned}
\|D C(x, y)\| & =\lim _{n \rightarrow \infty} \frac{1}{8^{n}}\left\|D f\left(2^{n} x, 2^{n} y\right)\right\| \\
& \leq \lim _{n \rightarrow \infty} \frac{1}{8^{n}} \psi\left(2^{n} x, 2^{n} y\right)=0
\end{aligned}
$$


for all $x \in X$. Therefore $C$ is a cubic mapping, which is unique. $\square$

Corollary 2.3. Let $p$ and $\lambda$ be non-negative real numbers such that $p<3$. Suppose that $f: X \rightarrow Y$ is a mapping satisfying

$$
\|D f(x, y)\| \leq \lambda\left(\|x\|^{p}+\|y\|^{p}\right)
$$

for all $x, y \in X$. Then, there exists a unique cubic mapping $C: X \rightarrow Y$ such that

$$
\|f(x)-C(x)\| \leq \frac{2^{p} \lambda}{2\left(8-2^{p}\right)}\|x\|^{p}
$$

for all $x \in X$.

Proof: The result follows from Theorem 2.2 by using $\psi(x, y)=\lambda\left(\|x\|^{p}+\|y\|^{p}\right)$.

Now, we establish the superstability of cubic mapping on Banach spaces.

Corollary 2.4. Let $p, q, \lambda$ be non-negative real numbers such that $p, q \in(3, \infty)$. Suppose a mapping $f: X \rightarrow Y$ satisfies

$$
\|D f(x, y)\| \leq \lambda\left\|x||^{q}\right\| y \|^{p}
$$

for all $x, y \in X$. Then, $f$ is a cubic mapping on $X$.

Proof: Letting $x=y=0$ in (2.9), we get $f(0)=0$. Once more, if we put $x=0$ in (2.9), we have $f(2 x)=8 f(x)$ for all $x \in X$. It is easy to see that by induction, we have $f\left(2^{n} x\right)=$ $8^{n} f(x)$, and so $f(x)=\frac{f\left(2^{n} x\right)}{8^{n}}$ for all $x \in X$ and $n \in \mathbb{N}$. Now, it follows from Theorem 2.2 that $f$ is a cubic mapping. $\square$

Note that in Corollary 2.4, if $p+q \in(0,3)$ and $p>0$ such that the inequality (2.9) holds, then by applying $\psi(x, y)=\lambda\|x\|^{p}\|y\|^{q}$ in Theorem $2.2, f$ is again a cubic mapping.

Theorem 2.5. Let $f: X \rightarrow Y$ be a mapping with $f(0)=0$, and let $\psi: X \times X \rightarrow[0, \infty)$ be a function satisfying

$$
\lim _{n \rightarrow \infty} 8^{n} \psi\left(\frac{x}{2^{n}}, \frac{y}{2^{n}}\right)=0
$$

and

$$
\|D f(x, y)\| \leq \psi(x, y)
$$

for all $x, y \in X$. If there exists $L \in(0,1)$ such that

$$
\psi(x, 0) \leq \frac{1}{8} L \psi(2 x, 0)
$$

for all $x \in X$, then there exists a unique cubic mapping $C: X \rightarrow Y$ such that

$$
\|f(x)-C(x)\| \leq \frac{L}{16(1-L)} \psi(x, 0)
$$

for all $x \in X$.

Proof: We consider the set $\Omega:=\{g: X \rightarrow Y \mid g(0)=0\}$ and introduce the generalized metric on $\Omega$ :

$$
d\left(g_{1}, g_{2}\right):=\inf \left\{C \in(0, \infty):\left\|g_{1}(x)-g_{2}(x)\right\| \leq C \psi(x, 0) \forall x \in X\right\}
$$

if there exists such constant $C$, and $d\left(g_{1}, g_{2}\right)=\infty$, otherwise. It is easy to show that $(\Omega, d)$ is complete. We will show that the mapping $\mathcal{J}: \Omega \rightarrow \Omega$ defined by 
$\mathcal{J} g(x)=8 g\left(\frac{x}{2}\right) ;(x \in X)$ is strictly contractive. For given $g_{1}, g_{2} \in \Omega$ such that $d\left(g_{1}, g_{2}\right)$

$<C$, we have

$$
\left\|8 g_{1}\left(\frac{x}{2}\right)-8 g_{2}\left(\frac{x}{2}\right)\right\| \leq \frac{1}{8} C \psi(2 x, 0)
$$

for all $x \in X$. By using (2.12), we obtain

$$
\left\|8 g_{1}\left(\frac{x}{2}\right)-8 g_{2}\left(\frac{x}{2}\right)\right\| \leq C L \psi(x, 0)
$$

for all $x \in X$. It follows from the last inequality that $d\left(\mathcal{J} g_{1}, \mathcal{J} g_{2}\right) \leq L d\left(g_{1}, g_{2}\right)$ for all $g_{1}, g_{2} \in \Omega$. Hence, $\mathcal{J}$ is a strictly contractive mapping on $\Omega$ with Lipschitz constant $L$. By putting $y=0$ and replacing $x$ by $\frac{x}{2}$ in (2.11) and using (2.12), then by dividing both sides of the resulting inequality by 2 , we have

$$
\left\|8 f\left(\frac{x}{2}\right)-f(x)\right\| \leq \frac{1}{2} \psi\left(\frac{x}{2}, 0\right) \leq \frac{1}{16} L \psi(x, 0)
$$

for all $x \in X$. Hence, $d(f, \mathcal{J} f) \leq \frac{L}{16}<\infty$. By applying the fixed point alternative, there exists a unique mapping $C: X \rightarrow Y$ in the set $\Omega_{1}=\{g \in \Omega$; $d(f, g)<\infty\}$ such that

$$
C(x)=\lim _{n \rightarrow \infty} 8^{n} f\left(\frac{x}{2^{n}}\right)
$$

for all $x \in X$. Again, Theorem 2.1 shows that

$$
d(f, C) \leq \frac{d(f, \mathcal{J} f)}{1-L} \leq \frac{L}{16(1-L)}
$$

where the inequality (2.15) implies the relation (2.13). Replacing $x, y$ by $2^{n} x, 2^{n} y$ in (2.11), respectively, and using (2.10) and (2.14), we conclude

$$
\begin{aligned}
\|D C(x, y)\| & =\lim _{n \rightarrow \infty} 8^{n}\left\|D f\left(\frac{x}{2^{n}}, \frac{y}{2^{n}}\right)\right\| \\
& \leq \lim _{n \rightarrow \infty} 8^{n} \psi\left(\frac{x}{2^{n}}, \frac{y}{2^{n}}\right)=0
\end{aligned}
$$

for all $x \in X$. Therefore $C$ is a cubic mapping. $\square$

Corollary 2.6. Let $p$ and $\lambda$ be non-negative real numbers such that $p>3$. Suppose that $f: X \rightarrow Y$ is a mapping satisfying

$$
\|D f(x, y)\| \leq \lambda\left(\|x\|^{p}+\|y\|^{p}\right)
$$

for all $x, y \in X$. Then there exists a unique cubic mapping $C: X \rightarrow Y$ such that

$$
\|f(x)-C(x)\| \leq \frac{\lambda}{2\left(2^{p}-8\right)}\|x\|^{p}
$$

for all $x \in X$.

Proof: It is enough to let $\psi(x, y)=\lambda\left(\|x\|^{p}+\|y\|^{p}\right)$ in Theorem 2.5. $\square$

Corollary 2.7. Let $p, q, \lambda$ be non-negative real numbers such that $p+q \in(0,3)$ and $p>0$. Suppose a mapping $f: X \rightarrow Y$ satisfies

$$
\|D f(x, y)\| \leq \lambda\|x \mid\|^{q}\|y\|^{p}
$$


for all $x, y \in X$. Then, $f$ is a cubic mapping on $X$.

Proof: If we put $x=y=0$ in (2.16), we get $f(0)=0$. Again, putting $x=0$ in (2.16), we conclude that $f(x)=8 f\left(\frac{x}{2}\right)$, and thus $f(x)=8^{n} f\left(\frac{x}{2^{n}}\right)$ for all $x \in X$ and $n \in \mathbb{N}$. Now, we can obtain the desired result by Theorem 2.5. $\square$

One should remember that if a mapping $f: X \rightarrow Y$ satisfies the inequality (2.16), where $p, q, \lambda$ be non-negative real numbers such that $p+q>3$ and $p>0$, then it is obvious that $f$ is a cubic mapping on $X$ by putting $\psi(x, y)=\lambda\|x\|^{p}\|y\|^{q}$ in Theorem 2.5.

\section{Stability of cubic multipliers}

In this section, we investigate the Hyers-Ulam stability and the superstability of cubic multipliers.

Definition 3.1. A cubic multiplier on an algebra $A$ is a cubic mapping $T: A \rightarrow A$ such that $a T(b)=T(a) b$ for all $a, b \in A$.

The following example introduces a cubic multiplier on Banach algebras.

Example. Let $(A,\|\cdot\|)$ be a Banach algebra. Then, we take $B=A \times A \times A \times A \times A \times A=$ $A^{6}$. Let $a=\left(a_{1}, a_{2}, a_{3}, a_{4}, a_{5}, a_{6}\right)$ be an arbitrary member of $B$ where we define \|\|$a\left\|\mid=\sum_{i=1}^{6}\right\| a_{i} \|$. It is easy to see $\left.(B,\|\| \cdot \| \mid)\right)$ is a Banach space. For two elements $a=$ $\left(a_{1}, a_{2}, a_{3}, a_{4}, a_{5}, a_{6}\right)$ and $b=\left(b_{1}, b_{2}, b_{3}, b_{4}, b_{5}, b_{6}\right)$ of $B$, we define $a b=\left(0, a_{1} b_{4}, a_{1} b_{5}+\right.$ $\left.a_{2} b_{6}, 0, a_{4} b_{6}, 0\right)$. It is easy to show that $B$ is a Banach algebra. We define $T: B \rightarrow B$ by $T$ $(a)=a^{3}$ for all $a \in B$. It is shown in Eshaghi Gordji et al. [16] that $T$ is a cubic multiplier on $A$.

Theorem 3.2. Let $f: A \rightarrow A$ be a mapping with $f(0)=0$ and let $\psi: A^{4} \rightarrow[0, \infty)$ be a function such that

$$
\|D f(x, y)+f(z) w-z f(w)\| \leq \psi(x, y, z, w)
$$

for all $x, y, z, w \in A$. If there exists a constant $L \in(0,1)$ such that

$$
\psi(2 x, 2 y, 2 z, 2 w) \leq 8 L \psi(x, y, z, w)
$$

for all $x, y, z, w \in A$, then there exists a unique cubic multiplier $T$ on A satisfying

$$
\|f(x)-T(x)\| \leq \frac{L}{2(1-L)} \psi(x, x, 0,0)
$$

for all $x \in A$.

Proof. It follows from the relation (3.2) that

$$
\lim _{n \rightarrow \infty} \frac{\psi\left(2^{n} x, 2^{n} y, 2^{n} z, 2^{n} w\right)}{8^{n}}=0
$$

for all $x, y, z, w \in A$.

Putting $y=z=w=0$ in (3.1), we obtain

$$
\|2 f(2 x)-16 f(x)\| \leq \psi(x, 0,0,0)
$$

for all $x \in A$. Thus,

$$
\left\|\frac{1}{8} f(2 x)-f(x)\right\| \leq \frac{1}{16} \psi(x, 0,0,0)
$$

for all $x \in A$. 
Now, similar to the proof of theorems in previous section, we consider the set $X:=$ $\{h: A \rightarrow A \mid h(0)=0\}$ and introduce the generalized metric on $X$ as:

$$
d\left(h_{1}, h_{2}\right):=\inf \left\{C \in \mathbb{R}^{+}:\left\|h_{1}(x)-h_{2}(x)\right\| \leq C \psi(x, 0,0,0) \text { for all } x \in A\right\}
$$

if there exists such constant $C$, and $d\left(h_{1}, h_{2}\right)=\infty$, otherwise. The metric space $(X, d)$ is complete, and by the same reasoning as in the proof of Theorem 2.2, the mapping $\Phi: X \rightarrow X$ defined by $\Phi(h)(x)=\frac{1}{8} h(2 x) ;(x \in A)$ is strictly contractive on $X$ and has a unique fixed point $T$ such that $\lim _{n \rightarrow \infty} d\left(\Phi^{n} f, T\right)=0$, i.e.,

$$
T(x)=\lim _{n \rightarrow \infty} \frac{f\left(2^{n} x\right)}{8^{n}}
$$

for all $x \in A$. By Theorem 2.1, we have

$$
d(f, T) \leq \frac{d(f, \Phi f)}{1-L} \leq \frac{L}{2(1-L)} .
$$

The proof of Theorem 2.2 shows that $T$ is a cubic mapping. If we substitute $z$ and $w$ by $2^{n} z$ and $2^{n} w$ in (3.1), respectively, and put $x=y=0$ and we divide the both sides of the obtained inequality by $2^{4 n}$, we get

$$
\left\|z \frac{f\left(2^{n} w\right)}{8^{n}}-\frac{f\left(2^{n} z\right)}{8^{n}} w\right\| \leq \frac{\psi\left(0,0,2^{n} z, 2^{n} w\right)}{2^{4 n}} .
$$

Passing to the limit as $n \rightarrow \infty$ and from (3.4), we conclude that $z T(w)=T(z) w$ for all $z, w \in A$.

Corollary 3.3. Let $r, \theta$ be non-negative real numbers with $r<3$ and let $f: A \rightarrow A$ be a mapping with $f(0)=0$ such that

$$
\|D f(x, y)+f(z) w-z f(w)\| \leq \theta\left(\left\|x||^{r}+\right\| y\left\|^{r}+\right\| z\left\|^{r}+\right\| w \|^{r}\right)
$$

for all $x, y, z, w \in A$. Then, there exists a unique cubic multiplier $T$ on $A$ satisfying

$$
\|f(x)-T(x)\| \leq \frac{2^{r-1} \theta}{8-2^{r}}\|x\|^{r}
$$

for all $x \in A$.

Proof. The proof follows from Theorem 3.2 by taking

$$
\psi(x, y, z, w)=\theta\left(\|x\|^{r}+\|y\|^{r}+\|z\|^{r}+\|w\|^{r}\right)
$$

for all $x, y, z, w \in A$. $\square$

Now, we have the following result for the superstability of cubic multipliers.

Corollary 3.4. Let $r_{j}(1 \leq j \leq 4)$. $\theta$ be non-negative real numbers with $\sum_{j=1}^{4} r_{j}<3$ and let $f: A \rightarrow A$ be a mapping with $f(0)=0$ such that

$$
\|D f(x, y)+f(z) w-z f(w)\| \leq \theta\left(\left\|x||^{r_{1}}\right\| y\left\|^{r_{2}}\right\| z\left\|^{r_{3}}|| w\right\|^{r_{4}}\right)
$$

for all $x, y, z, w \in A$. Then, $f$ is a cubic multiplier on $A$.

Proof. It is enough to let $\psi(x, y, z, w)=\theta\left(\left.\left\|x||^{r_{1}}\right\| y\left\|\left.\right|^{r_{2}}\right\| z\left\|^{r_{3}}\right\| w\right|^{r_{4}}\right)$ in Theorem 3.2. $\square$ 


\section{Competing interests}

The authors declare that they have no competing interests.

\section{Authors' contributions}

The work presented here was carried out in collaboration between all authors. AB suggested to write the current paper. All authors read and approved the final manuscript.

\section{Author details}

${ }^{1}$ Department of Mathematics, Garmsar Branch, Islamic Azad University, Garmsar, Iran ${ }^{2}$ Institute for Mathematical

Research, University Putra Malaysia, 43400 Upm, Serdang, Selangor Darul Ehsan, Malaysia

Received: 21 March 2011 Accepted: 13 September 2011 Published: 13 September 2011

\section{References}

1. Ulam, SM: Problems in Modern Mathematics. Wiley, New York, sciencechapt. VI (1940)

2. Hyers, DH: On the stability of the linear functional equation. Proc Natl Acad Sci. 27, 222-224 (1941). doi:10.1073/ pnas.27.4.222

3. Rassias, ThM: On the stability of the linear mapping in Banach spaces. Proc Am Math Soc. 72, 297-300 (1978). doi:10.1090/S0002-9939-1978-0507327-1

4. Găvruta, P: A generalization of the Hyers-Ulam-Rassias stability of approximately additive mappings. J Math Anal Appl 184, 431-436 (1994). MR1281518 (95e:47089). doi:10.1006/jmaa.1994.1211

5. Skof, F: Propriet locali e approssimazione di operatori. Rend Sem Math Fis Milano. 53, 113-129 (1983). doi:10.1007/ BF02924890

6. Cholewa, PW: Remarks on the stability of functional equations. Aequ Math. 27, 76-86 (1984). doi:10.1007/BF02192660

7. Czerwik, S: On the stability of the quadratic mapping in normed spaces. Abh Math Sem Univ Hamburg 62, 59-64 (1992). MR1182841 (94e:39026). doi:10.1007/BF02941618

8. Eshaghi Gordji, M, Bodaghi, A: On the Hyers-Ulam-Rasias stability problem for quadratic functional equations. East Journal of Approximations. 16(2), 123-130 (2010)

9. Eshaghi Gordji, M, Bodaghi, A: On the stability of quadratic double centralizers on Banach algebras. J Comput Anal Appl. 13(4), 724-729 (2011)

10. Lee, J, An, J, Park, C: On the stability of qudratic functional equations. Abstr Appl Anal. 2008, 1-9 (2008)

11. Jun, KW, Kim, HM: The generalized Hyers-Ulam-Rassias stability of a cubic functional equation. J Math Anal Appl. 274 867-878 (2002). doi:10.1016/S0022-247X(02)00415-8

12. Jun, KW, Kim, HM: On the Hyers-Ulam-Rassias stability of a general cubic functional equation. Math Inequal Appl. 6(2), 289-302 (2003)

13. Chang, IS, Jun, KW, Jung, YS: The modified Hyers-Ulam-Rassias stability of a cubic type functional equation. Math Inequal Appl. 8(4), 675-683 (2005)

14. Najati, A: The generalized Hyers-Ulam-Rassias stability of a cubic functional equation. Turk J Math. 31, 395-408 (2007)

15. Bodaghi, A, Alias, IA, Eshaghi Gordji, M: On the stability of quadratic double centralizers and quadratic multipliers: a fixed point approach. J Inequal Appl. 2011(Article ID 957541), 9 (2011)

16. Eshaghi Gordji, M, Shams, S, Ramezani, M, Ebadian, A: Approximately cubic double centralizers. Nonlinear Func Anal Appl. 15(3), 503-512 (2010)

17. Turinici, M: Sequentially iterative processes and applications to Volterra functional equations. Ann Univ Mariae-Curie Sklodowska (Sect A). 32, 127-134 (1978)

18. Diaz, JB, Margolis, B: A fixed point theorem of the alternative for contractions on a generalized complete metric space. Bull Am Math Soc. 74, 305-309 (1968). doi:10.1090/S0002-9904-1968-11933-0

doi:10.1186/1029-242X-2011-53

Cite this article as: Bodaghi et al:: Approximately cubic functional equations and cubic multipliers. Journal of Inequalities and Applications 2011 2011:53.

\section{Submit your manuscript to a SpringerOpen ${ }^{\circ}$ journal and benefit from:}

- Convenient online submission

- Rigorous peer review

- Immediate publication on acceptance

- Open access: articles freely available online

- High visibility within the field

- Retaining the copyright to your article

Submit your next manuscript at $\mathbf{s p r i n g e r o p e n . c o m ~}$ 